\title{
Design Flow of English Learning System Based on Item Response Theory
}

\author{
https://doi.org/10.3991/ijet.v12.i12.7958 \\ Yuemei Liu $\left({ }^{\bowtie}\right)$, Xuetao Zhao \\ Hebei Agricultural University, Baoding, China \\ liuyuemei19821126@163.com
}

\begin{abstract}
The popularity of computer technology in English teaching has led to the establishment of many English learning platforms, but the enhancement of students' English proficiency is limited due to the lack of relevance, self-adaptive test questions and analytical ability. The project management theory is introduced into English learning, which can provide students with teaching content and test questions that are more suitable for their own actual situation through a more intelligent, personalized way. At the same time, the static and dynamic database model based on students' own learning behavior is constructed to facilitate storage of students' learning record. Combined with the advantages of hierarchical selection, SH method and improved polynomial model, this paper puts forward a new type of item section model. This paper introduces the basic theory and related technology, and then makes an in-depth study on the demand analysis of English learning system. Finally, this paper realizes the design of English learning system based on item response theory and validates the good effect of English item selection from the perspective of application. The system provides teachers and students with convenient learning strategies, item selection strategies, test strategies and academic performance strategies. The introduction of item response theory enables the system to become truly student-centered and provides a more comprehensive and selfadaptive learning model, which is of great significance for improving the learning efficiency of English learning and the learning efficiency of college students in China.
\end{abstract}

Keywords-item response theory, English learning, data model, item selection model, system requirements

\section{Introduction}

The rapid development of Internet technology and computer technology has pushed the process of network information modernization. Computer network technology has permeated into various fields and is widely applied in English teaching field. The English learning platform and system designed and created with the help of computer network technology have improved the English proficiency of students in China. However, the shortcomings like the lack of relevance, self-adaptive test questions and analytical ability can be found in most systems [1]. In view of the shortcomings 
of previous systems, this paper introduces the item response into the design of English learning platforms, and builds a student-centered system, which achieves the selfadaptive selection of corresponding learning and test content according to the English ability, proficiency and knowledge of students [2].

The key point of this paper is to propose a new item selection strategy, which combines the advantages of hierarchical selection, SH method and improved polynomial model. This strategy gives consideration to both width and precision of the test, and provides English learners of different levels with the most suitable items [3]. This paper first introduces the item response theory, ability parameter estimation technique and item selection strategy, and proposes a new selection strategy [4]. Then the business process and demand analysis of the system are introduced, determining the design goal and the overall design framework of the system. The development environment of the system is described in the implementation phase of the system, and the main modules of the system are developed. The system interface of students' personalized learning and student ability test is demonstrated through screenshots.

The English learning system based on the item response theory can intelligently adjust the learning content in the next phase according to the mastery of students [5], which helps students to know their own English learning proficiency in a better way; at the same time, the system can conduct parameter setting on learn strategies, item selection strategies, test strategies and academic performance strategies, which facilitates teachers to achieve teaching management. The design and implementation of the system have important application significance to improve the efficiency and management of English learning.

\section{$2 \quad$ Basic theory and key technology}

\subsection{Item response theory}

In order to provide theoretical guidance for the development and selection of test items, the item response theory (IRT) is introduced, which is a theory related to the psychological test that evaluates the competency of testees [6]. The ability of the testees includes the mastery of knowledge, comprehensive ability and answering ability. Item response theory can eliminate the error brought by single measurement through multiple tests so that the ability of testees can be summarized and handled scientifically. Moreover, through the potential characteristics of the testees, the project characteristic curve can be built and then the test items can be screened according to this curve.

The potential characteristic is a psychological feature of testees and does not exist in the human body in the form of material. These psychological features are independent of each other, and the set of these potential features is called the potential feature space, which can be expressed as, where is a component of a potential feature. The introduction of item response theory has the following three advantages for the examination system: 
1. Correct the measurement error index and improve the adaptability and measurement accuracy;

2. The parameter estimation is independent, unacted on samples, and the parameter estimation accuracy is improved;

3. The information function modeling improves the test efficiency;

4. It has a comparable test scheme.

The item response model can be divided into one-dimensional model and multidimensional model, and the dimension is proportional to the number of potential features [7]. The logic model is widely applied in the project characteristic mathematical model and it can be divided into single parameter model, two-parameter model and three-parameter model according to the number of parameters.

\subsection{Ability parameter estimation technology}

The key of the English learning system is that the computer can provide learning resources and self-adaptive learning content and processes according to the students' ability. The self-adaptive tests provided by the computer can be divided into two phases: the initial assessment phase of the students' ability and the accurate assessment phase of the students' ability.

The algorithms used in initial assessment phase and the accurate assessment phase are shown in Table 1:

Table 1. The two phases used algorithmic comparisons

\begin{tabular}{|c|c|c|}
\hline Phases & Algorithm name & Description \\
\hline \multirow{3}{*}{$\begin{array}{l}\text { Initial assessment } \\
\text { phase }\end{array}$} & Intermediate value method & $\begin{array}{l}\text { Extraction of medium difficulty topics, if pass, } \\
\text { increase the difficulty }\end{array}$ \\
\hline & $\begin{array}{l}\text { Historical information assessment } \\
\text { method }\end{array}$ & $\begin{array}{l}\text { Calculate the level through historical perfor- } \\
\text { mance }\end{array}$ \\
\hline & Self-selected method & Select the initial difficulty by own \\
\hline \multirow{2}{*}{$\begin{array}{c}\text { Accurate assessment } \\
\text { phase }\end{array}$} & Maximum Likelihood Estimatio & $\begin{array}{l}\text { Build data response matrix, use mathematical } \\
\text { operation to obtain the ability value }\end{array}$ \\
\hline & $\begin{array}{l}\text { Bayesian expectation posteriori } \\
\text { estimation method }\end{array}$ & Obtain by Bayesian theorem \\
\hline
\end{tabular}

\subsection{Modified item selection strategy}

Only when the items are suitable for the level of the testees can they improve the ability of them. At present, the application strategies that are commonly used include similar selection method, maximum information selection method and hierarchical selection method. These strategies have their own characteristics, but have their own shortcomings, such as unable to control the exposure rate, content balance. Therefore, it is necessary to make improvements [8].

The improved item selection strategy proposed in this paper mainly solves the problem of the balance between the exposure rate and the content of the items. The calculation method of distribution index of exposure rate [9]: 


$$
\mathrm{X}^{2}=\sum_{\mathrm{j}=1}^{\mathrm{N}} \frac{\left(\mathrm{er}_{\mathrm{j}}-\mathrm{L} / \mathrm{N}\right)^{2}}{\mathrm{~L} / \mathrm{N}}
$$

refers to the exposure rate of the jth item in the application process. Exposure index is inversely proportional to the utilization of the items.

The improvement is conducted on the basis of $\mathrm{C}$ hierarchical selection method. The main idea of $\mathrm{C}$ layer selection method is: firstly, divide the item pool into different groups in accordance with the content of items, and then divide each group into different item blocks according to the difficulty. After that, arrange the items with small differentiation from each item region in the same hierarchy. Repeat these processes and finally the hierarchical selection structure is formed [10]. Finally, combine the same hierarchy in each group into a sub-item bank. The polynomial model and SympsonHetter algorithm are introduced to solve the problem of exposure rate and balance. The specific process of the improved item strategy is as follows:

1. Set the maximum exposure rate within the range of $0.2-0.3$ and set the maximum exposure rate of the jth project as.

2. Through the SympsonHetter iteration of each item, obtain the exposure parameter value. The method of simulation test is adopted. The probability of being selected for the jth project is $\mathrm{P}()$ and the implementation probability is $\mathrm{P}$ If $\mathrm{P}$, then the exposure rate of this project is ; or else the parameter is 1 (Modarresi, G. et al., 2011).

3. Produce cumulative target ratio distribution, and the target ratio of 1.0; divide the item banks into different groups according to content and the number of groups is G.

4. Arrange each item from easy to difficult according to the difficulty value $b$ in the item response model.

5. Conduct the difficulty partition within the group. Allocate the items with the lowest degree of difficulty in the first region and the items in the last partition has the highest degree of difficulty.

6. The items inare sorted in ascending order according to differentiation degree a.

7. Divide the items in into $\mathrm{K}$ hierarchies.

8. The $\mathrm{K}$ layers in all groups are merged to form $\mathrm{k}$ hierarchies.

9. Adjust the test process to $\mathrm{K}$ hierarchies and match it with the $\mathrm{K}$ hierarchies in the item bank.

10. Use the polynomial model method to process each test phase. For the Kth phase, select the coefficient of difficulty that matches the current content in the Kth layer. In the item response model calculation, obtain the test items that are closest to the testees.

11. The exposure rate is controlled by a random number that follows the uniform distribution $U(0,1)$. If the exposure rate of the selected item is greater than the random number, the item is implemented; otherwise, the next round of judgment is conducted.

12. Repeat steps 10 to 11 until the test is completed. 


\section{Demands analysis and design of the English learning system}

\subsection{Analysis of demands for English learning system}

\section{Business process analysis.}

Obtain English Learning Resources. List-based English management is to provide learners with the audios, videos, electronic documents and other resources in the form of a list through the network platform on which learners can download materials to learn or just learn online after choosing learning contents. List-based English learning resource management, though user-friendly, cannot offer students targeted resource screening, failing to meet the self-adaptive goals [11].

However, this English teaching platform, based on the level of learning, the recent test scores, learners' learning objectives, or an exercise test, can master the users' learning conditions, thus providing targeted English learning resources.

Practice and Evaluation Business Processes. Exercises and tests are the main means to achieve effective teaching management and learning feedbacks. Both exercises and tests require individualized teaching based on the level of learners. The item response theory introduced in the previous chapter plays an important role in the process of selecting exercises and exam questions. By choosing adaptive questions, we can promote the enthusiasm of learning and understand our failure in knowledge [12-13].

System Configuration Business Process. The front end provides the humancomputer interaction interface, which meets the needs of teachers for teaching management and students for English learning. The back-end part is mainly for the storage and preservation of students' learning records and the archiving and database storage of learning materials.

Teaching Management Business Process. The scope of teaching management includes system management, school teaching management, and student management, and the system platform should realize the communication between the three and realtime information exchange.

System functional requirements analysis. System functional requirements summarize the requirements of the users and managers of the system for the system's functions. The roles in English learning system are mainly students, teachers, teaching managers, and system managers [14].

Figure 1 shows the main functional requirements of students.

After students log in the system with a separate user name and password, the system can provide students with learning resources based on their own English level who can study independently in the personalized learning home page. The practice and test module can provide materials covering grammar, spoken, listening, reading, etc. The topic selection strategy based on the item response theory shall be able to scientifically and rationally guide the study, and recommend the relevant learning resources.

Figure 2 shows the main functional requirements of the teacher participants.

Teachers are able to post and update the teaching resources through the system and upload new test questions to help students learn independently. In order to better 
correct test questions with high error rate, the system needs to provide teachers with error statistical information to help teachers with learning diagnosis, or to enable teachers to accept students' feedbacks and reply to student questions in the teaching process.

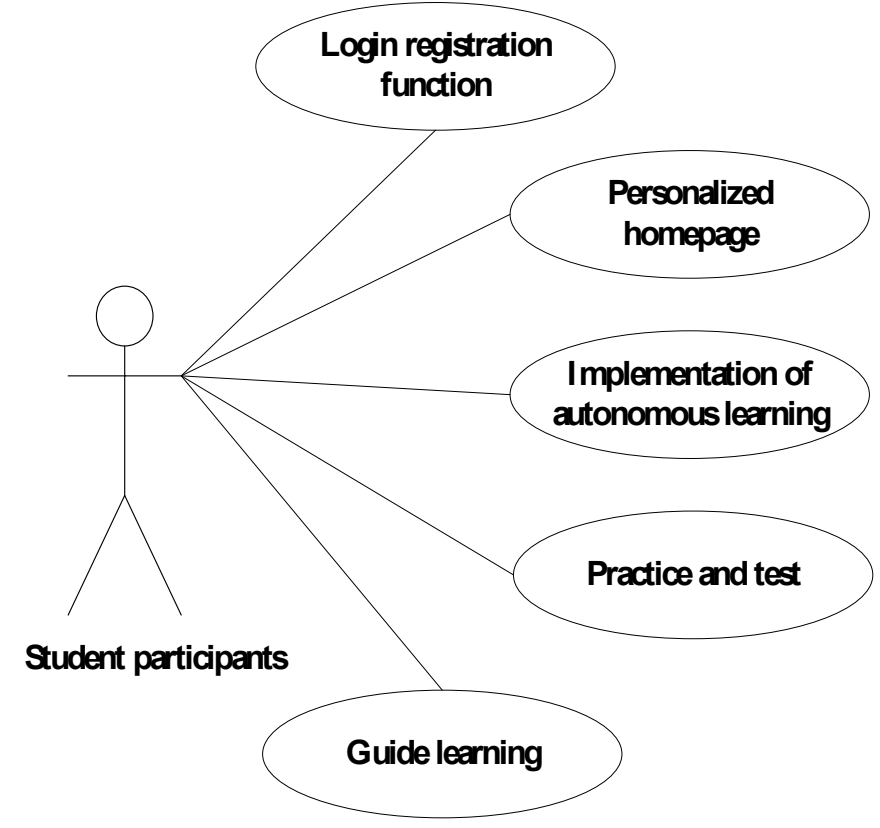

Fig. 1. The main functional requirements of student participants

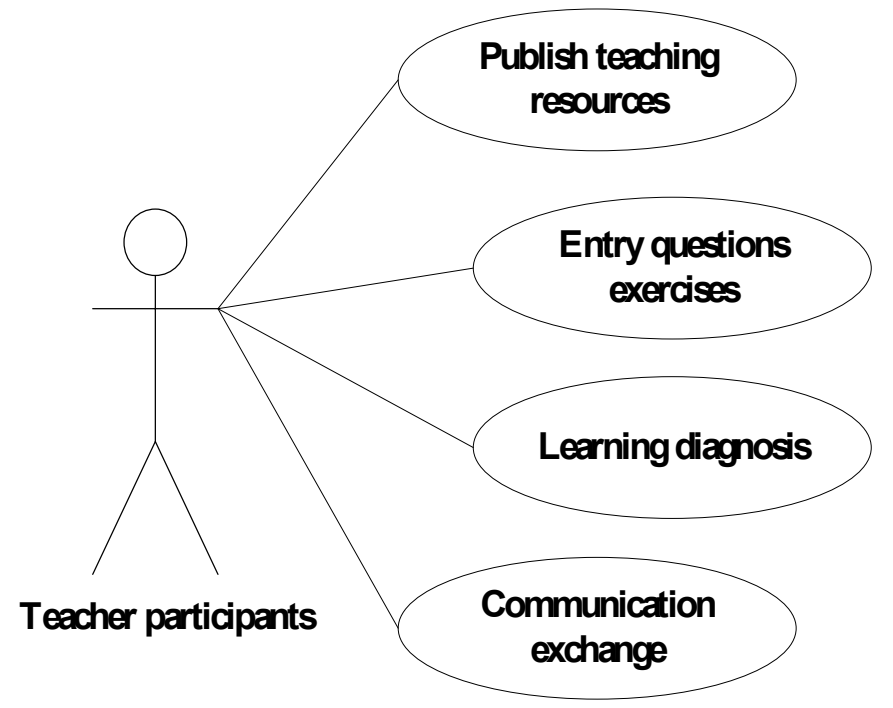

Fig. 2. The main functional requirements of teacher participants 
Teaching managers are mainly responsible for the management of teaching strategies, model parameters, student user management, test parameter configuration, and other memory management. Management staff tends to emphasis on the management and maintenance of the system itself, with their demands covering school user management, communication and exchange security, database management, and overall system configuration parameters.

Analysis of system performance requirements. Practicability and simple and convenient operations can save time for users except in English study. The stability enables the system good performance without dead machine or response failure, so as to ensure the application effect in real practice. The extensibility enables the system to be expanded under the overall structure to meet the requirements of new functional modules [15].

\subsection{Design of English learning system}

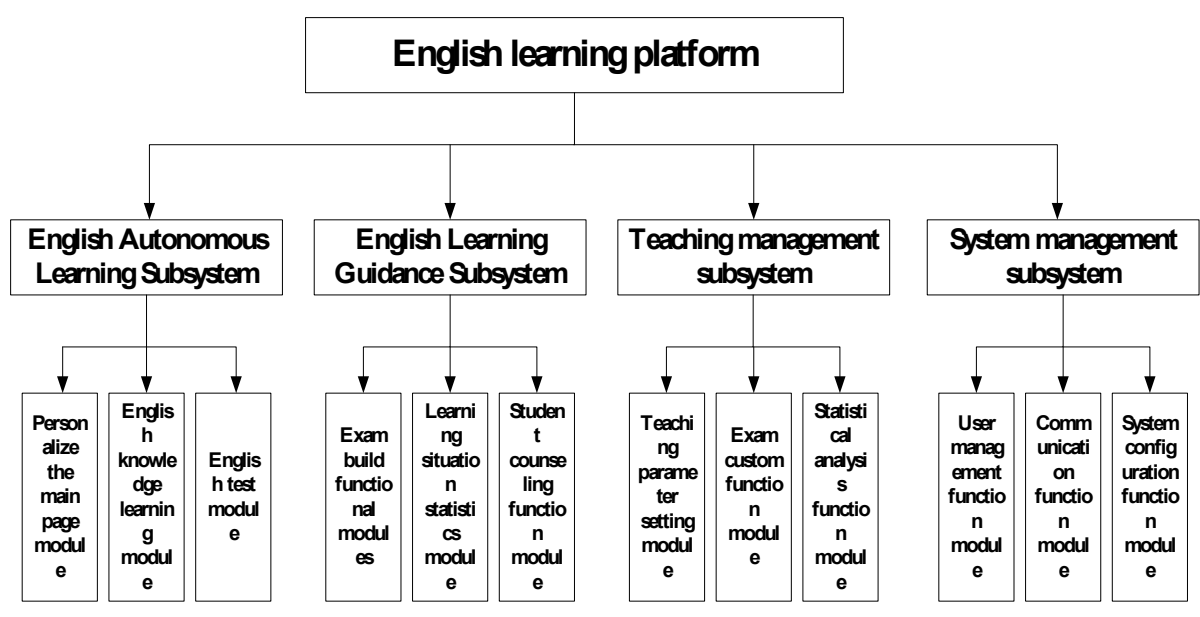

Fig. 3. English learning system function diagram

Overall system architecture design. The overall architecture of the system mainly includes the functional architecture and logical architecture of the system, based on the integration of the design objectives of the English learning system and the requirements of the system. The overall architecture of the system can be divided into the English independent learning subsystem, the English learning instruction subsystem, the teaching management subsystem, and the system management subsystem, respectively for students, teachers, school administrators, and system managers, 4 kinds of users of the system. The architecture diagram of the system is shown in figure 3.

Each of the four subsystems has the corresponding function to meet the needs of different users. Each sub-module of the human-computer interaction interface is independent of each other, but they can share data and realize real-time interaction in the back end. 
Logic architecture design of the system. The functional architecture of the English learning system analyzes and designs the various subsystems and functional modules of the platform based on the users' perspectives. The logical architectural design of the system is shown in figure 4.

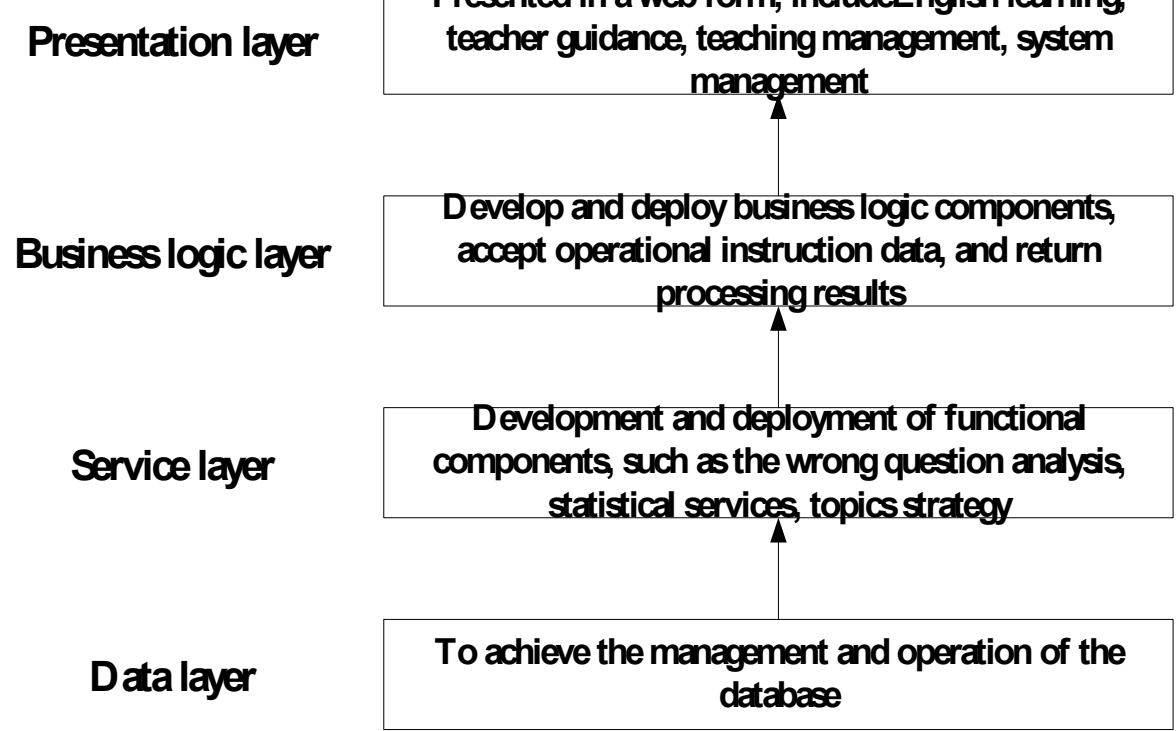

Fig. 4. System logic architecture design

The system adopts the layered architecture consisting of the presentation layer, business logic layer, service layer and data layer to realize the functions of each function module.

The presentation layer is the interface between the system platform and users, and the business logic layer accepts user data and instructions from the presentation layer. Then the service layer is to provide basic function for the business logic layer, while the data layer is mainly to realize the data access and management for different types of databases.

System database design. Taking the structure of the English item database table structure as an example, the database structure adopted by system data access is described. The English item data table is mainly used to store the English exercises and items based on the theory of item response. The structure of the data table design is shown in Table 2.

Each ID field is the number of each English item, and Store ID is used to identify the item stored in the item bank; the data table also designs three other fields, which is used by teachers to set three parameters of items.

The data storage structure of student test results statistics table, school information table, student learning record chart are similar to item data, which will not be described in detail. 
Table 2. English test database structure

\begin{tabular}{|c|c|c|c|c|}
\hline Serial number & Field ID & Field Type & Field length & Remarks \\
\hline 1 & ID & int & 4 & English title number \\
\hline 2 & Store ID & int & 4 & Exam ID \\
\hline 3 & Type ID & int & 4 & Type ID \\
\hline 4 & Topic Content & nText & null & Topic Content \\
\hline 5 & Answer & nChar & 100 & Answer \\
\hline 6 & Difficulty & double & 8 & Difficulty \\
\hline 7 & Discrimination & double & 8 & Discrimination \\
\hline 8 & Guess & double & 8 & Guess \\
\hline
\end{tabular}

\section{The realization of English learning system}

\subsection{Introduction to system development environment}

The system is developed by using Silverlight technology, which is part of the framework of Microsoft's .Net Framework platform. This technology completes the system interface development, business logic development, and service development by C \# language, with Windows 7 operating platform as the system's software development platform and Microsoft SQL Server 2010 database management system as the database.

The development software and platform that develop the system are both Microsoft products for their mutual compatibility, improving the system development efficiency and ensuring the operations of the English learning system.

\subsection{Implementation and interface display of system function modules}

Implementation and interface display of students personalized page. Students personalized interface includes the error summary of the current student user, the learning history, learning diagnostic reports, personal information, etc. Personalized learning services are designed to, based on practical ability, learning level, and learning objectives of students, develop a reasonable learning program, and record and analyze the learning performance, so as to make teaching more effective. Figure 5 is the interface to analyze students' ability.

As shown in the figure, it refers to the personalized learning interface under the students learning subsystem, in which students can perform listening, reading, grammar, translation, reading exercises and tests. Based on students' historical test scores, the system will score different aspects of the user's ability by percentage for students to know their own English level. At the same time, the system will show points of all users in the individual ranking list of the system, as a way to effectively encourage students to learn independently.

Students competency testing interface. The core of the student ability test function is the realization of the strategy algorithm. According to the new topic selection strategy proposed in Chapter 2, the system can test the student's ability by the item response theory. Figure 6 is the student's listening ability test interface. If students can 
Paper-Design Flow of English Learning System Based on Item Response Theory

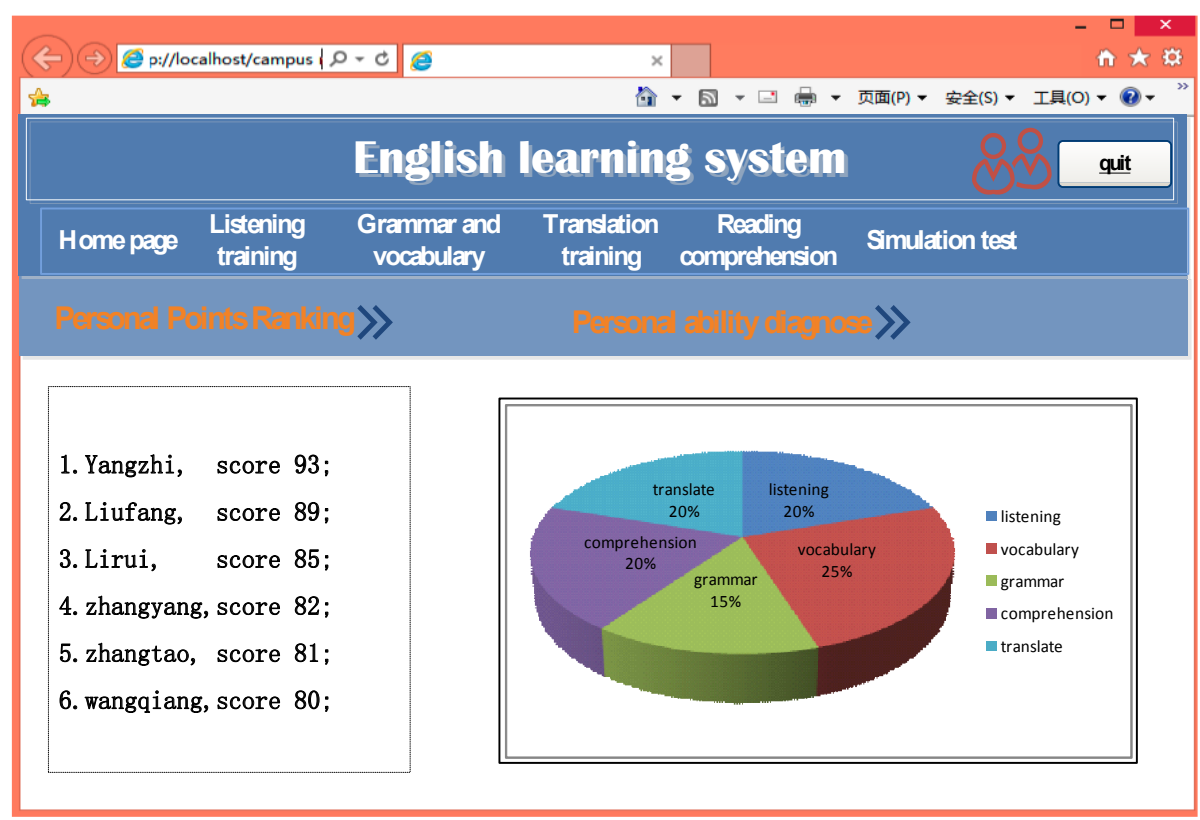

Fig. 5. The Students personalized page interface map

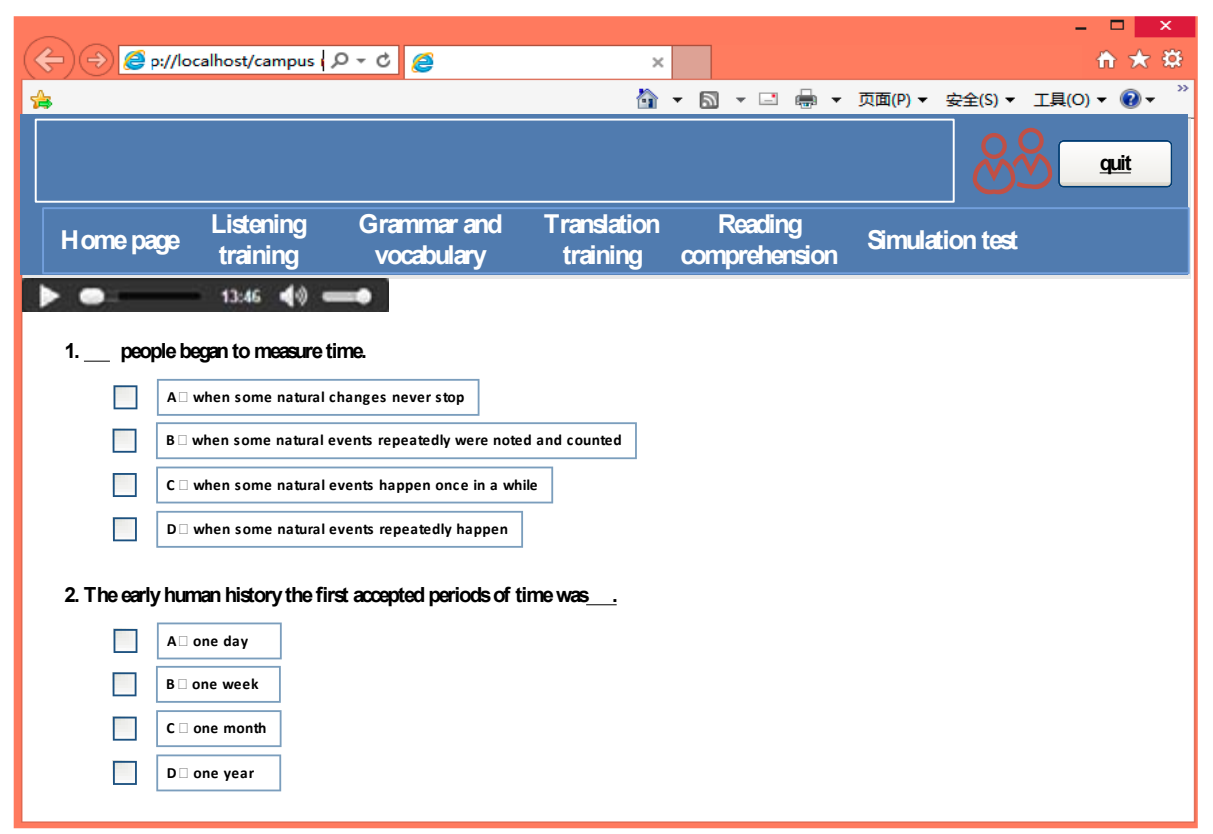

Fig. 6. Student listening ability test interface 
complete the listening test within the specified time, the system can assess the listening ability level of students based on students' scores.

The system offers reading, grammar, and translations tests to students similar to listening ability tests. The new topic selection strategy can provide students with more suitable learning contents which will stimulate students' interests in learning and improve students' efficiency.

\section{Conclusion}

The English learning system designed in this paper has both intelligent and adaptive characteristics. The introduction of the item response theory enables the system to provide personalized services for students and teachers. The new topic selection strategy is the core for the system to provide students with adaptive English learning materials. The main contents and significance of this paper are as follows:

1. The introduction of the item response theory, capability parameter estimation technology and topic selection strategy provide the theoretical basis for the design of the system.

2. The users' functional requirements and the overall framework of the system were analyzed and designed.

3. This paper designed the English learning system based on the item response theory, and displayed the interface of the core function sub-modules of the system.

4. The design of the system not only facilitates the implementation and control of teaching, but also helps with the teaching-system integrated management and improves the intelligent level of the system.

\section{References}

[1] Zhu, L., Xing, W. (2011). The analysis of English network computer aid test system model and technology selection. IEEE, 1-4.

[2] Ma, L., Yu, X. (2011). Concepts of constructing college English autonomous learning network system. Energy Procedia, 13: 10264-10268.

[3] Schmitt, S. (2002). Simvar: A similarity-influenced question selection criterion for e-sales dialogs. Artificial Intelligence Review, 18(3-4): 195-221. https://doi.org/10.1023/A:10207 45614238

[4] Wei, W., Xie, Q., Yao, S. (1995). A kinetic parameter estimation technique with long path length spectroelectrochemical method. Electrochimica Acta, 40(8): 1057-1061. https://doi.org/10.1016/0013-4686(93)E0009-B

[5] Linden, W.J.V.D., Hambleton, R.K. (1996). Handbook of modern item response theory. Journal of the American Statistical Association, 92(439): 245-256.

[6] Hays, R.D., Morales, L.S., Reise, S.P. (2000). Item response theory and health outcomes measurement in the 21st century. Medical Care, 38(9 Supply): II28.

[7] Reise, S.P., Widaman, K.F., Pugh, R.H. (1993). Confirmatory factor analysis and item response theory: Two approaches for exploring measurement invariance. Psychological Bulletin, 114(3): 552-66. https://doi.org/10.1037/0033-2909.114.3.552 
[8] Ziehn, T., Scholze, M., Knorr, W. (2012). On the capability of monte Carlo and adjoint inversion techniques to derive posterior parameter uncertainties in terrestrial ecosystem models. Global Biogeochemical Cycles, 26(3): 3025. https://doi.org/10.1029/2011GB0041 $\underline{85}$

[9] Linden, W.J.V.D. (2003). Some alternatives to sympson-hetter item-exposure control in computerized adaptive testing. Journal of Educational \& Behavioral Statistics, 28(3): 249265. https://doi.org/10.3102/10769986028003249

[10] Leung, C.K., Chang, H.H., Hau, K.T. (2002). Item selection in computerized adaptive testing: improving the a-stratified design with the sympson-hetter algorithm. Applied Psychological Measurement, 26(4): 376-392. https://doi.org/10.1177/014662102237795

[11] Modarresi, G., Rahbar, M., Bateni, B., Ojaqi, M., Sheikhzadehmarand, E. (2011). An analysis of demotivating factors in learning English. Virus Research, 160(1-2): 326.

[12] Hsu, M.H. (2008). A personalized English learning recommender system for ESL students. Expert Systems with Applications, 34(1): 683-688. https://doi.org/10.1016/j.eswa.2006.10. $\underline{004}$

[13] Sun, C.Y., Chang, K.Y. (2016). Design and development of a location-based mobile learning system to facilitate English learning. Universal Access in the Information Society, 15(3): 1-13. https://doi.org/10.1007/s10209-014-0392-x

[14] Li, Q. (2017). Design and realization of project-based computer English learning system. International Journal of Emerging Technologies in Learning, 12(8): 128. https://doi.org/10.3991/ijet.v12i08.7147

[15] Gao, H., Ma, Y., Geng, P. (2017). Design and implementation of English learning system based on computer network platform. International Journal of Emerging Technologies in Learning, 12(8): 77. https://doi.org/10.3991/ijet.v12i08.7139

\section{$7 \quad$ Authors}

Yuemei Liu is with the College of foreign language, Hebei Agricultural University, Baoding 071001, China.

Xuetao Zhao is with the College of foreign language, Hebei Agricultural University, Baoding 071001, China.

Article submitted 09 November 2017. Published as resubmitted by the authors 12 December 2017. 